\title{
Developing a Mammalian Behaviour Ontology
}

\section{Introduction}

The EuroPhenome mouse phenotyping resource (http://www.europhenome.org) ${ }^{1}$ is the central depository for raw phenotype data generated from carrying out the standard operating procedures (SOPs) defined for the EUMODIC high-throughput mouse phenotyping project. An ontology annotation framework is implemented within EuroPhenome which makes use of the PATO Entity and Quality (EQ) model and the species-specific Mammalian Phenotype (MP) ontology to apply 4-levels of annotation: SOP annotation; parameter annotation; qualitative data annotation; and the automatic annotation of mutant lines ${ }^{2}$. This annotation framework standardises the input, storage and analysis of data across all SOPs.

An OBO Foundry-compliant ontology which defines the mammalian behaviour phenotype domain does not currently exist. The species-neutral Animal Behaviour Ontology $(A B O)^{3}$ distinguishes between the ground facts about what an animal does (e.g. running, biting, vocalizing) and the functions attributed by human domain experts to the direct behaviour observations (e.g. prey capture, assertion of dominance, guarding of territory). In its current form the granularity of terms within $A B O$ are too coarse for the description of mouse behaviours. Therefore a dedicated behaviour ontology is required to define mammalian behaviours at a useful resolution.

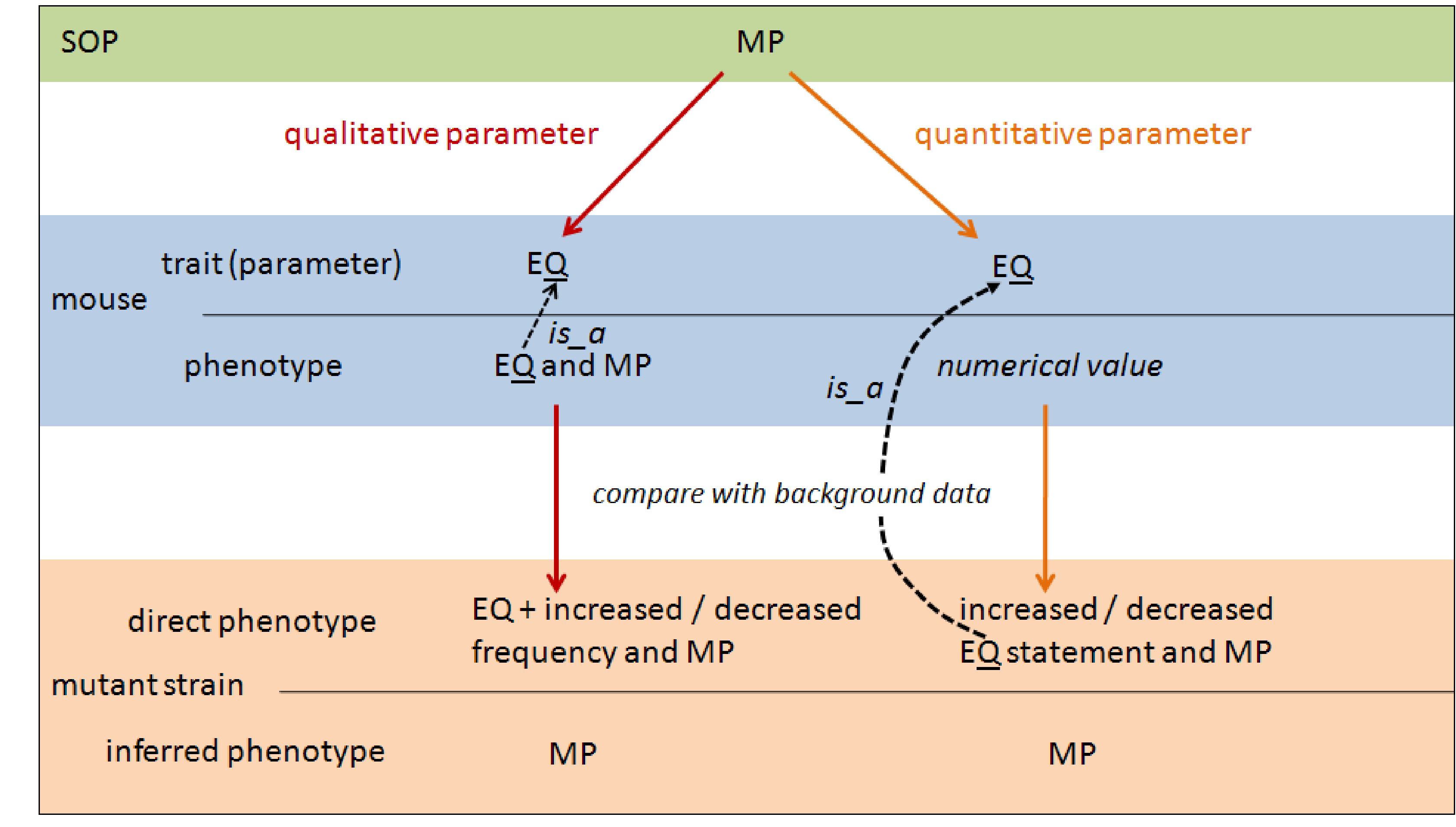

Levels of ontology annotation of mouse phenotype data within EuroPhenome. $\mathrm{MP}=$ Mammalian Phenotype ontology. $\mathrm{EQ}=$ Entity + Quality.

\section{Design rationale}

The Mammalian Behaviour Ontology (MBO) is being developed to precisely define mammalian behaviours, while maintaining the behavioural activity and behavioural function distinction defined by $A B O$. This distinction forms the two top-level classes of the ontology. The MBO also draws a fundamental distinction between behavioural activities and functions in isolation from other individuals and behaviours between individuals, which are broadly analogous to the Gene Ontology biological process concepts "adult behaviour" and "behavioral interaction between organisms". Subsequent child classes are currently in the process of being manually added. Here we present a snapshot of the current upper-level structure of the MBO.

The $\mathrm{MBO}$ is being developed in line with OBO Foundry guidelines and will shortly be made publicly available for OBO community scrutiny.

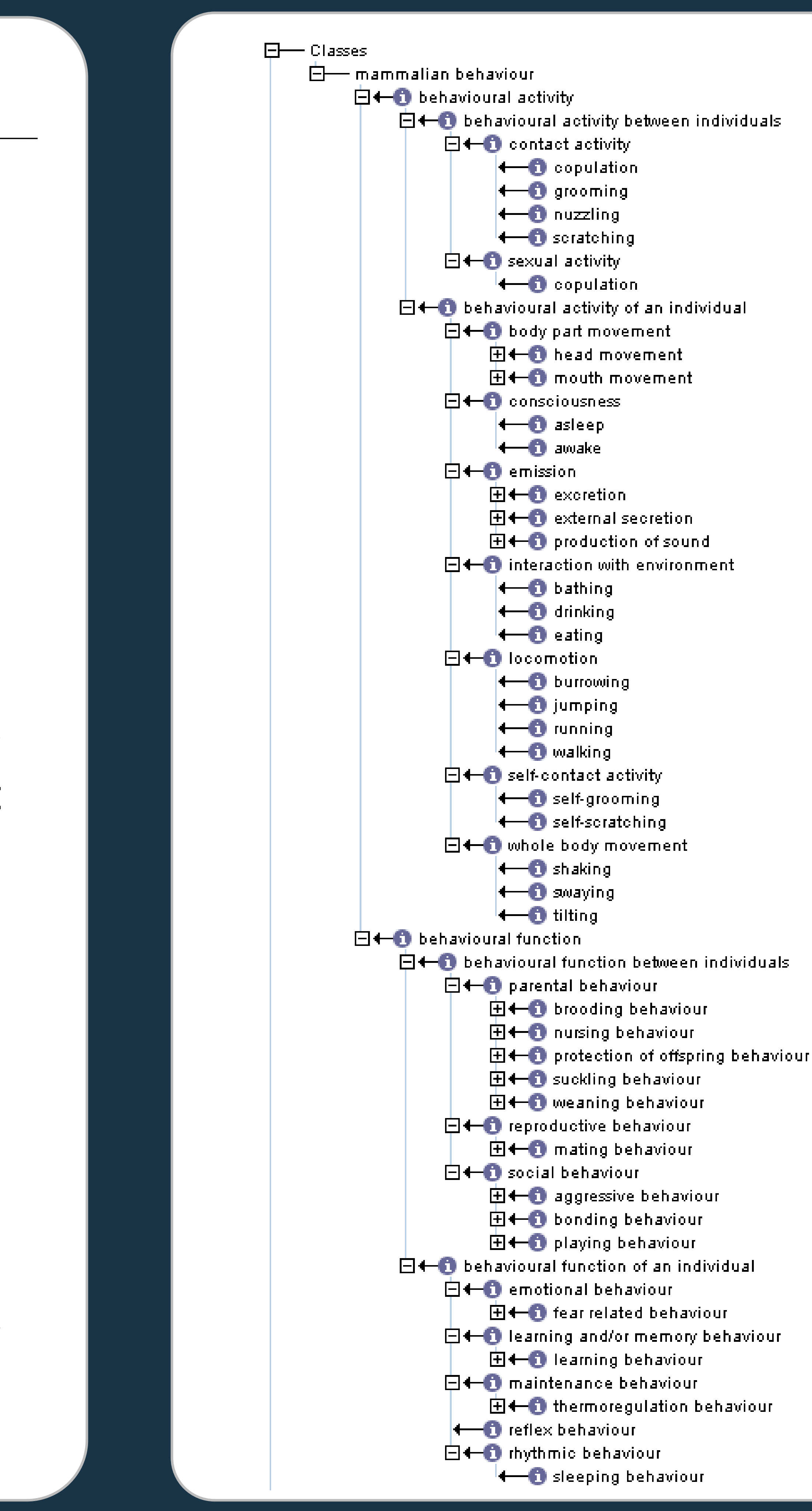

\section{Term population and use}

As a minimum criteria set for the MBO term population, the MBO will endeavor to contain all phenotypes currently defined within the "behavior/neurological phenotype" branch of MP; the behavioral phenotypes observed during the execution of the EUMODIC behavioural SOPs; the behaviours relevant to mammals contained within $A B O$ and within the "behavior" branch of GO; and the phenotypes defined in the Glossary of Rat Behaviours (http://www.ratbehavior.org/Glossary.htm).

In the short-term the MBO will be an ontology of mouse and rat behaviours. It will be implemented within EuroPhenome in the composition of EQ annotations. Concepts from the MBO will assist the construction of the behavioural branch of the emerging vertebrate Trait Ontology. We anticipate the further development of the MBO to span the behaviours of other mammals, chiefly human, thus facilitating mouse model to human disease associations.

\section{Summary}

The Mammalian Behaviour Ontology is needed in order to provide unambiguous descriptions of behaviours which can be used in the postcomposed EQ representation of phenotypes. A current challenge in the description of phenotypes is distinguishing between the directly observed phenotype and the inferred phenotype, with additional biological meaning, made after expert analysis. The MBO makes this distinction with the categorisation of phenotypes as either direct behavioural activities or inferred behavioural functions.

This process of developing the MBO will rely on contributions from experts from within both the animal behaviour and ontology communities. Please register your interest with getting involved in this project by e-mailing: t.beck@har.mrc.ac.uk.

\section{References and Acknowledgements}

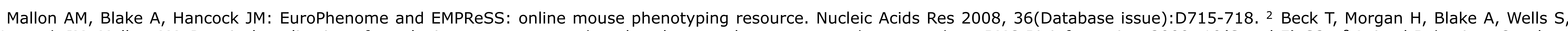

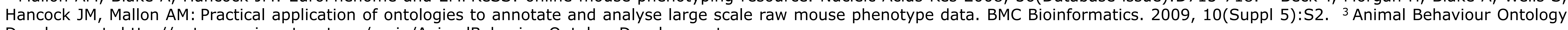
Development: http://ontogenesis.ontonet.org/moin/AnimalBehaviourOntologyDevelopment. 